\title{
Six-Degree-of-Freedom Digital Simulations for Missile Guidance and Control
}

\author{
Yongjie Xu, Zhijun Wang, and Benbing Gao \\ College of Mechatronic Engineering, North University of China, Taiyuan 030051, China \\ Correspondence should be addressed to Yongjie Xu; yongqiang515@126.com
}

Received 17 March 2015; Accepted 25 June 2015

Academic Editor: Roman Lewandowski

Copyright (C) 2015 Yongjie Xu et al. This is an open access article distributed under the Creative Commons Attribution License, which permits unrestricted use, distribution, and reproduction in any medium, provided the original work is properly cited.

Six-degree-of-freedom ballistic trajectory model can be used to verify design indicators, performance parameters, and correctness so they have been put forward in the missile argumentation and design process. Mathematical simulation is the basis of late semiphysical simulation and field firing testing, and it can greatly reduce the field shooting test time, which shortens the missile test cycle and so reduces costs.

\section{Introduction}

In the course of the development of precision-guided missiles, a large number of experiments were used to assess the performance of the missile and verify that the performance index in the process of missile design meets requirements whether or not [1-3]. Cost of range test experiment is high and cycle is long; therefore, method of mathematical simulation is adopted as usual in the early project development stage of missile guided weapons. After building skid-to-turn (STT) missile six-degree-of-freedom ballistic trajectory simulation model which is composed of important parts of the model, such as dynamic model, control system model, and guidance system model, it can be used to evaluate missile operational effectiveness [4-6].

\section{Missile Control System Design}

Missile control system design is generally conducted in complex domain: first, transferring time domain differential equation model of missile to transfer function model in complex domain by Laplace transformation and then designing PID controller based on model characteristics to realize control for missile system. Controller parameters obtained according to missile aerodynamic derivatives, for pitch channel, yaw channel and rolling channel, respectively, are obtained by single channel dynamic simplified model design [7].

\subsection{Single Channel Dispersion Equation and Transfer}

Functions Model

2.1.1. Simplified Dynamic Model of Missile. Generally for airto-air missile, we use simplified conditions as follows:

(1) Solidification principle: at a certain moment $t$ in ballistic trajectory, speed $V$, flying height $H$, and engine thrust $P$, the mass of the missile $m$ and moment of inertia $j$ are changeless.

(2) Missile has an axisymmetric layout form.

(3) Missile parameters change a little and the attack angle is smaller when missile is under control and interference.

(4) Control system guarantees stability rolling angle and sufficient rapidity.

The rigid-body six-degree-of-freedom nonlinear differential equations are time domain equations and are formed by five-part series equations (1)-(5) as follows: 


$$
\begin{aligned}
& m \frac{d V}{d t}=P \cos \alpha \cos \beta-X-m g \sin \theta \\
& m V \frac{d \theta}{d t}=P\left(\sin \alpha \cos \gamma_{V}+\cos \alpha \sin \beta \sin \gamma_{V}\right)+Y \cos \gamma_{V}-Z \sin \gamma_{V}-m g \cos \theta \\
& -m V \cos \theta \frac{d \varphi_{V}}{d t}=P\left(\sin \alpha \sin \gamma_{V}-\cos \alpha \sin \beta \cos \gamma_{V}\right)+Y \sin \gamma_{V}+Z \cos \gamma_{V}, \\
& J_{x} \frac{d \omega_{x}}{d t}+\left(J_{z}-J_{y}\right) \omega_{z} \omega_{y}=M_{x} \\
& J_{y} \frac{d \omega_{y}}{d t}+\left(J_{x}-J_{z}\right) \omega_{x} \omega_{z}=M_{y} \\
& J_{z} \frac{d \omega_{z}}{d t}+\left(J_{y}-J_{x}\right) \omega_{y} \omega_{x}=M_{z} \\
& \frac{d x}{d t}=V \cos \theta \cos \varphi_{V} \\
& \frac{d y}{d t}=V \sin \theta \\
& \frac{d z}{d t}=-V \cos \theta \sin \varphi_{V} \\
& \frac{d \vartheta}{d t}=\omega_{y} \sin \gamma+\omega_{z} \cos \gamma \\
& \frac{d \varphi}{d t}=\frac{1}{\cos \vartheta}\left(\omega_{y} \cos \gamma-\omega_{z} \sin \gamma\right) \\
& \frac{d \gamma}{d t}=\omega_{x}-\tan \vartheta\left(\omega_{y} \cos \gamma-\omega_{z} \sin \gamma\right) \\
& \sin \beta=\cos \theta\left[\cos \gamma \sin \left(\varphi-\varphi_{V}\right)+\sin \vartheta \sin \gamma \cos \left(\varphi-\varphi_{V}\right)\right]-\sin \theta \cos \vartheta \sin \gamma, \\
& \sin \alpha=\frac{\left\{\cos \theta\left[\sin \vartheta \cos \gamma \cos \left(\varphi-\varphi_{V}\right)-\sin \gamma \sin \left(\varphi-\varphi_{V}\right)\right]-\sin \theta \cos \vartheta \cos \gamma\right\}}{\cos \beta}, \\
& \sin \varphi_{V}=\frac{(\cos \alpha \sin \beta \sin \vartheta-\sin \alpha \sin \beta \cos \gamma \cos \vartheta+\cos \beta \sin \gamma \cos \vartheta)}{\cos \theta} .
\end{aligned}
$$

The definitions of each parameter are as follows: $m$ : mass of missile, $P$ : thrust of engine, $X$ : aerodynamic drag, $Y$ : aerodynamic lift, $Z$ : aerodynamic lateral force, $g$ : gravitational acceleration, $V$ : velocity of missile, $\theta$ : trajectory inclination angle, $\alpha$ : attack angle, $\beta$ : sideslip angle, $\vartheta$ : pitching angle, $\varphi$ : yaw angle, $\gamma$ : roll angle, $\delta$ rudder angle, $\varphi_{V}$ : trajectory deflection angle, $\gamma_{V}$ : angle of velocity inclination, $J_{x}, J_{y}, J_{z}$ : rotational inertia component of coordinate axes, and $M_{x}, M_{y}$, $M_{z}$ : torque component of coordinate axes.

Introduce small perturbation assumption, ignore second order or higher order traces and secondary factors of aerodynamic forces and moment, linearize the equations, remove independent solved equations (3), and develop the missile perturbation equation in three-dimensional space:

$$
\begin{aligned}
m \frac{d \Delta V}{d t}= & \left(P^{V}-X^{V}\right) \Delta V-\left(P^{\alpha}+X^{\alpha}\right) \Delta \alpha \\
& -G \cos \theta \Delta \theta, \\
m V \frac{d \Delta \theta}{d t}= & \left(P^{V} \alpha+Y^{V}\right) \Delta V+\left(P+Y^{\alpha}\right) \Delta \alpha \\
& +G \sin \theta \Delta \theta+Y^{\delta_{\vartheta}},
\end{aligned}
$$




$$
\begin{aligned}
& -m V \cos \theta \frac{d \Delta \varphi_{V}}{d t}=\left(-P+Z^{\beta}\right) \Delta \beta+\left(P^{\alpha}+Y\right) \Delta \gamma_{V} \\
& +Z^{\delta_{\varphi}} \Delta \delta_{\varphi} \\
& J_{x} \frac{d \Delta \omega_{x}}{d t}=M_{x}^{\beta} \Delta \beta+M_{x}^{\omega_{x}} \Delta \omega_{x}+M_{x}^{\omega_{y}} \Delta \omega_{y} \\
& +M_{x}^{\delta_{\gamma}} \Delta \delta_{\gamma}+M_{x}^{\delta_{\varphi}} \Delta \delta_{\varphi} \\
& J_{y} \frac{d \Delta \omega_{y}}{d t}=M_{y}^{\beta} \Delta \beta+M_{y}^{\omega_{x}} \Delta \omega_{x}+M_{y}^{\omega_{y}} \Delta \omega_{y} \\
& +M_{y}^{\dot{\beta}} \Delta \dot{\beta}+M_{y}^{\delta_{\varphi}} \Delta \delta_{\varphi} \\
& J_{z} \frac{d \Delta \omega_{z}}{d t}=M_{z}^{V} \Delta V+M_{z}^{\alpha} \Delta \alpha+M_{z}^{\omega_{z}} \Delta \omega_{z} \\
& +M_{z}^{\dot{\alpha}} \Delta \dot{\alpha}+M_{z}^{\delta_{\varphi}} \Delta \delta_{\varphi} \\
& \frac{d \Delta \vartheta}{d t}=\Delta \omega_{z} \\
& \frac{d \Delta \varphi}{d t}=\frac{1}{\cos \vartheta} \Delta \omega_{y} \\
& \frac{d \Delta \gamma}{d t}=\Delta \omega_{x 1}-\tan \vartheta \Delta \omega_{y}, \\
& \Delta \theta=\Delta \vartheta-\Delta \alpha, \\
& \Delta \varphi_{V}=\Delta \varphi+\frac{\alpha}{\cos \theta} \Delta \gamma-\frac{1}{\cos \theta} \Delta \beta, \\
& \Delta \gamma_{V}=\tan \theta \Delta \beta+\frac{\cos \varphi}{\cos \theta} \Delta \gamma .
\end{aligned}
$$

For axisymmetric missiles, rolling motion parameters are trace compared with longitudinal motion parameters; then, the three-dimensional equations of motion can be decomposed into three channels of motion differential equations:

$$
\begin{aligned}
\frac{d \Delta V}{d t}= & \frac{P^{V}-X^{V}}{m} \Delta V-\frac{P^{\alpha}-X^{\alpha}}{m} \Delta \alpha-g \cos \theta \Delta \theta, \\
\frac{d \Delta \theta}{d t}= & \frac{P^{V} \alpha-Y^{V}}{m V} \Delta V+\frac{P+Y^{\alpha}}{m V} \Delta \alpha+\frac{g \sin \theta}{V} \Delta \theta \\
& +\frac{Y^{\delta_{\vartheta}}}{m V} \Delta \delta_{\vartheta}, \\
\frac{d \Delta \omega_{z}}{d t}= & \frac{M_{z}^{V}}{J_{z}} \Delta V+\frac{M_{z}^{\alpha}}{J_{z}} \Delta \alpha+\frac{M_{z}^{\omega}}{J_{z}} \Delta \omega_{z}+\frac{M_{z}^{\dot{\alpha}}}{J_{z}} \Delta \dot{\alpha} \\
& +\frac{M_{z}^{\delta_{\vartheta}}}{J_{z}} \Delta \delta_{\vartheta}, \\
\frac{d \Delta \vartheta}{d t}= & \Delta \omega_{z}, \\
\Delta \theta= & \Delta \vartheta-\Delta \alpha .
\end{aligned}
$$

Then rigid-body longitudinal channel dynamic model (8) can be derived by (1)-(7) based on the simplified conditions:

$$
\begin{aligned}
\ddot{\vartheta}+a_{1} \dot{\vartheta}+a_{1}^{\prime} \dot{\alpha}+a_{2} \alpha+a_{3} \delta_{\vartheta} & =0, \\
\dot{\vartheta}+a_{4}^{\prime} \theta-a_{4} \alpha-a_{5} \delta_{\vartheta} & =0, \\
\vartheta-\theta-\alpha & =0 .
\end{aligned}
$$

In a similar way, rigid-body rolling channel dynamic model (9) can also be derived:

$$
\ddot{\gamma}+c_{1} \dot{\gamma}+c_{3} \delta_{\gamma}+c_{2} \beta+c_{4} \delta_{\varphi}=0 \text {. }
$$

Meaning of dynamic coefficients in rigid-body longitudinal channel and rolling channel is as follows.

$a_{1}=-M_{z}^{\omega_{z}} / J_{z}$ is missile aerodynamic damping coefficient. It is angular velocity increment caused by a unit increment of angle acceleration.

$a_{1}^{\prime}=M_{z}^{\dot{\alpha}} / J_{z}=\left(m_{z}^{\dot{\alpha}} q S L / J_{z}\right)(L / V)$ is an ignorable trace and it stands for the effect of downwash flow delay on missile pitching moment.

$a_{2}=-M_{z}^{\alpha} / J_{z}$ is coefficient of static stability and it characterizes the static stability of missile.

$a_{3}=-M_{z}^{\delta} / J_{z}$ is missile rudder efficiency coefficient. It is missile angular acceleration caused by a unit increment of control surface deflection.

$a_{4}=\left(Y^{\alpha}+P\right) / m V$ is angular velocity increment of ballistic tangent rotation.

$a_{4}^{\prime}=(-g / V) \sin \theta$ is an ignorable trace.

$a_{5}=Y^{\delta} / \mathrm{mV}$ is ballistic tangent rotation angular velocity increment caused by the control surface deflection, a unit when attack angle stays with no change.

$c_{1}=-M_{z}^{\omega_{z}} / J_{x}=\left(-m_{z}^{\omega_{z}} q S L / J_{x}\right)(L / 2 V)$ is missile aerodynamic damping coefficient in rolling direction.

$c_{2}=M_{x}^{\beta} / J_{x}$ is an ignorable trace.

$c_{3}=-M_{x}^{\delta_{x}} / J_{x}=-m_{x}^{\delta_{x}} q S L / J_{x}$ is missile aileron efficiency.

$c_{4}=-M_{x}^{\delta_{\varphi}} / J_{x}$ is an ignorable trace.

$a_{2}(\alpha)=\left(-57.3 C_{N}^{\alpha} q S L / J_{x}\right)\left(\left(x_{T}-x_{d}\right) / L\right)$ is angle of attack function, its status as positive or negative reflects static stability of the missile. It changes as the angle of attack angle missile static stability changes.

$S$ is the reference area, and generally $S$ is the maximum cross-section area of missile.

$L$ is the reference length, and generally $L$ is the diameter of missile. 


\subsubsection{Transfer Function}

(1) Pitch Channel Transfer Function. Laplace transform is used to transform rigid-body motion equations (8) under zero initial condition; that is,

$$
\begin{aligned}
s\left(s+a_{1}\right) \vartheta+\left(a_{1}^{\prime} s+a_{2}\right) \alpha & =-a_{3} \delta_{\vartheta}, \\
-a_{4} \alpha+\left(s+a_{4}^{\prime}\right) \theta & =a_{5} \delta_{\vartheta}, \\
\vartheta-\theta-\alpha & =0 .
\end{aligned}
$$

Parameter $s$ is a complex variable, derived from Laplace transformation.

Solve $\vartheta, \alpha, \theta$ through Cramer's Rule, and then (11)-(14) can be derived:

$$
\begin{aligned}
& \frac{\vartheta(s)}{\delta_{9}(s)}=-\frac{\left(a_{3}-a_{1}^{\prime} a_{5}\right) s+a_{3}\left(a_{4}+a_{4}^{\prime}\right)-a_{2} a_{5}}{s^{3}+c_{11} s^{2}+c_{22} s+c_{33}}, \\
& \frac{\theta(s)}{\delta_{9}(s)}=-\frac{-a_{5} s^{2}-a_{5}\left(a_{1}+a_{1}^{\prime}\right) s+a_{3} a_{4}-a_{2} a_{5}}{s^{3}+c_{11} s^{2}+c_{22} s+c_{33}}, \\
& \frac{\alpha(s)}{\delta_{9}(s)}=-\frac{a_{5} s^{2}+\left(a_{3}+a_{1} a_{5}\right) s+a_{3} a_{4}^{\prime}}{s^{3}+c_{11} s^{2}+c_{22} s+c_{33}} .
\end{aligned}
$$

Expression of each parameter is as follows:

$$
\begin{aligned}
& c_{11}=a_{1}+a_{3}+a_{4}^{\prime}+a_{1}^{\prime}, \\
& c_{22}=a_{2}+a_{1}\left(a_{4}+a_{4}^{\prime}\right)+a_{1}^{\prime} a_{4}^{\prime}, \\
& c_{33}=a_{2} a_{4}^{\prime} .
\end{aligned}
$$

The denominator of each equation is cubic polynomial, and its root is commonly a real root and a pair of conjugate complex root, namely:

$$
s^{3}+c_{11} s^{2}+c_{22} s+c_{33}=\left(s-\lambda_{1}\right)\left(s^{2}+B_{1} s+B_{2}\right) .
$$

By the perturbation theory, the second part of a conjugate complex root stands for oscillation motion, namely:

$$
s^{2}+B_{1} s+B_{2}=s^{2}+2 \varepsilon_{D} \omega_{D} s+\omega_{D}^{2} .
$$

Expression of each parameter is as follows:

$\varepsilon_{D}$ : natural frequency of rigid-body disturbance motion,

$\omega_{D}$ : damping coefficient of the rigid motion.

In view of the pitch channel, introduce time constant $T_{D}=1 / \omega_{D}$, and then

$$
\frac{\vartheta(s)}{\delta_{\vartheta}(s)}=\frac{K_{D}}{\left(T_{D}^{2} s^{2}+2 \varepsilon_{D} \omega_{D} s+1\right)} \cdot \frac{1}{s-\lambda_{1}} \cdot\left(T_{1 D} s+1\right) .
$$

Expression of each parameter is as follows:

$$
\begin{aligned}
& T_{1 D}=\frac{a_{3}-a_{1}^{\prime} a_{5}}{K_{D} \omega_{D}^{2}}, \\
& K_{D}=\frac{a_{3}\left(a_{4}+a_{4}^{\prime}\right)-a_{2} a_{5}}{\omega_{D}^{2}} .
\end{aligned}
$$

Ignore trace $\alpha_{1}^{\prime}, \alpha_{4}^{\prime}$, for winged missile $\alpha_{5} \approx 0$, and then

$$
\frac{\vartheta(s)}{\delta_{\vartheta}(s)}=\frac{K_{D}\left(T_{1 D} s+1\right)}{s\left(T_{D}^{2} s^{2}+2 \varepsilon_{D} T_{D} s+1\right)} .
$$

Then pitch transfer function (18) with rudder angle as input and pitching angle velocity as output can be derived by (10)-(17):

$$
\frac{\dot{\vartheta}(s)}{\delta_{\vartheta}(s)}=\frac{K_{D}\left(T_{1 D} s+1\right)}{T_{D}^{2} s^{2}+2 \varepsilon_{D} T_{D} s+1} .
$$

In a similar way, transfer function (19) can be derived:

$$
\begin{aligned}
& \frac{\dot{\theta}(s)}{\delta_{\vartheta}(s)}=\frac{K_{D}}{T_{D}^{2} s^{2}+2 \varepsilon_{D} T_{D} s+1}, \\
& \frac{n_{y}(s)}{\delta_{\vartheta}(s)}=\frac{V}{57.3 g} \cdot \frac{K_{D}}{T_{D}^{2} s^{2}+2 \varepsilon_{D} \omega_{D} s+1} .
\end{aligned}
$$

Expression of each parameter is as follows:

$$
\begin{aligned}
K_{D} & =\frac{a_{3} a_{4}}{a_{2}+a_{1} a_{4}}, \\
T_{1 D} & =\frac{1}{a_{4}}, \\
T_{D} & =\frac{1}{\sqrt{a_{2}+a_{1} a_{4}}}, \\
\varepsilon_{D} & =\frac{a_{1}+a_{4}}{2 \sqrt{a_{2}+a_{1} a_{4}}} .
\end{aligned}
$$

In order to express clearly, in the full equation control system, choose missile single channel motion model functional form with rudder declination as input and pitch angle velocity as output. For axisymmetric missile, its aerodynamic parameters of pitching motion are consistent with yaw motion.

(2) Rolling Channel Transfer Function. Ignore trace $c_{2}, c_{4}$ and rolling motion disturbance equation (9) can be expressed as

$$
\ddot{\gamma}+c_{1} \dot{\gamma}=-c_{3} \delta_{\gamma} .
$$

Laplace transform is used to transform rolling motion disturbance equation under zero initial condition, and then

$$
s\left(s+c_{1}\right) \gamma(s)=-c_{3} \delta_{\gamma}(s) .
$$

The following equation can be derived:

$$
\frac{\gamma(s)}{\delta_{\gamma}(s)}=\frac{K_{D X}}{s\left(T_{D X} s+1\right)} .
$$

Then rolling channel transfer function (24) with rudder angle as input and roll angle velocity as output can be derived by $(21)-(23)$ :

$$
\frac{\dot{\gamma}(s)}{\delta_{\gamma}(s)}=\frac{K_{D X}}{T_{D X} s+1} .
$$

Expression of each parameter is as follows:

$$
\begin{aligned}
& K_{D X}=-c_{3} / c_{1}: \text { roll motion transfer coefficient, } \\
& T_{D X}=1 / c_{1}: \text { roll motion time constant. }
\end{aligned}
$$


2.1.3. Simplified Rigid-Body Motion Model. (1) Consider longitudinal rigid motion equation of normal type layout missile:

$$
\begin{aligned}
& \frac{\dot{\vartheta}(s)}{\delta_{\vartheta}(s)}=\frac{K_{D}\left(T_{1 D} s+1\right)}{T_{D}^{2} s^{2}+2 \varepsilon_{D} T_{D} s+1}, \\
& \frac{\dot{\theta}(s)}{\delta_{\vartheta}(s)}=\frac{K_{D}}{T_{D}^{2} s^{2}+2 \varepsilon_{D} T_{D} s+1} .
\end{aligned}
$$

(2) Consider the whole moving missile wing type layout or canard configuration type layout missile longitudinal rigid motion equation:

$$
\begin{aligned}
& \frac{\dot{\vartheta}(s)}{\delta_{\vartheta}(s)}=-\frac{K_{D}\left(T_{q D} s+1\right)}{T_{D}^{2} s^{2}+2 T_{D} \zeta_{D} s+1}, \\
& \frac{\dot{\theta}(s)}{\delta_{\vartheta}(s)}=-\frac{K_{D}\left(T_{\theta}^{2} s^{2}+2 T_{\theta} \zeta_{\theta} s+1\right)}{T_{D}^{2} s^{2}+2 T_{D} \zeta_{D} s+1} .
\end{aligned}
$$

Expression of each parameter is as follows:

$$
\begin{aligned}
K_{D} & =\frac{\left|a_{3}\right| a_{4}+a_{2}(\alpha) a_{5}}{a_{1} a_{4}+a_{2}(\alpha)}, \\
T_{q D} & =\frac{\left|a_{3}\right|+a_{1}^{\prime}(\alpha) a_{5}}{\left|a_{3}\right| a_{4}+a_{2}(\alpha) a_{5}}, \\
K_{D}^{2} & =\frac{1}{a_{1} a_{4}+a_{2}(\alpha)}, \\
2 T_{D} \zeta_{D} & =\frac{a_{1}+a_{1}^{\prime}(\alpha) a_{5}+a_{4}}{a_{1} a_{4}+a_{2}(\alpha)}, \\
T_{D}^{2} & =\frac{a_{5}}{\left|a_{3}\right| a_{4}+a_{2}(\alpha) a_{5}}, \\
2 T_{\theta} \zeta_{\theta} & =\frac{\left[a_{1}+a_{1}^{\prime}(\alpha)\right] a_{5}}{\left|a_{3}\right| a_{4}+a_{2}(\alpha) a_{5}} .
\end{aligned}
$$

(3) The rolling motion equation of missile is expressed as

$$
\frac{\dot{\gamma}(s)}{\delta_{\gamma}(s)}=\frac{K_{D X}}{T_{D X} s+1} .
$$

\subsection{PID Controller Design}

2.2.1. PID Controller Parameter Setting. The PID controller parameter setting is the core content of control system design. It determines PID controller proportionality coefficient and integral and differential time size according to the character of charged process.

There are a lot of methods for PID controller parameter setting; in summary there are two broad categories: one is the theoretical calculation setting method. It depends mainly on the basis of mathematical model of the system and determines the controller parameters through theory calculation. Another is the engineering setting method; it mainly depends on engineering experience and is directly used in experiment of control system; it is simple, easy to master, and widely used in engineering practice.

Engineering setting method of PID controller parameters mainly has the critical ratio method, reaction curve method, and decay method. The three methods have their characteristics; their common ground is through test, and then the engineering experience formula is used to set controller parameters $[8,9]$.

Now the critical ratio method is commonly used. Using the method of PID controller parameter setting, the steps are as follows: (1) for the first preliminary choose a short enough sampling period to make the system work; (2) only join proportional control link, until the system step response of critical oscillation emerges, and write down the proportion amplification coefficient and the critical oscillation cycle; (3) calculate the parameters of PID controller under a certain degree of control by the formula.

2.2.2. Principle of PID Parameter Setting. $P, I, D$ parameters preset is complementary to each other; fine tuning should be done according to the actual conditions as follows: if the physical quantity controlled oscillation is near the target, first, increase the integral time $I$ and if there is still an oscillation, we can reduce the proportional gain $P$ appropriately. If the physical quantity controlled is difficult to recover after change, first, increase the proportional gain $P$, and if the recovery is still relatively slow, we can appropriately reduce integration time $I$ and still can increase the differential time D.

2.3. Controller Parameters Design. Missile control system adopts adjustable parameters strategy and control system adjustable parameters including inner loop gain coefficient $K$ inner and outer loop PI controller parameters $K_{p}, K_{i}$. In the control system design progress, according to a set of typical missile flight status, first, the missile's aerodynamic parameters are obtained by wind tunnel test; obtain linear model of missile by the aerodynamic parameters and then design adjustable parameter $K_{\text {inner, }} K_{p}, K_{i}$, separately according to the linear model; in the actual flight process when missile flies in typical condition, determine the value of adjustable parameter $K_{\text {_inner, }} K_{i}, K_{p}$, according to the interpolation algorithm $[10,11]$.

2.3.1. Structure of Missile Control System. The overall structure of the missile control system is shown in Figure 1.

Control system is composed of pitch channel, yaw channel, and rolling channel; the system input is the overload of pitch and yaw in the normal direction and rolling angle of rolling shaft. System output is angle of the rudder of each channel to the missile dynamic module, attitude angle, angle of attack and velocity, angular velocity, and other information that can be obtained using the missile dynamics module and feedback loop control system made up of missile angular velocity and acceleration information.

(1) Pitch Channel Autopilot. Pitch channel circuit is the main channel for controlling missile mobile stably and fast under 


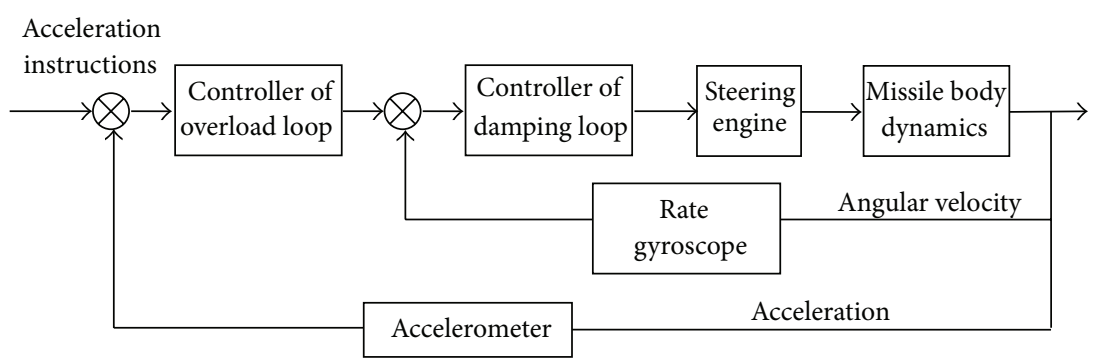

FIGURE 1: Missile control system overall structure.

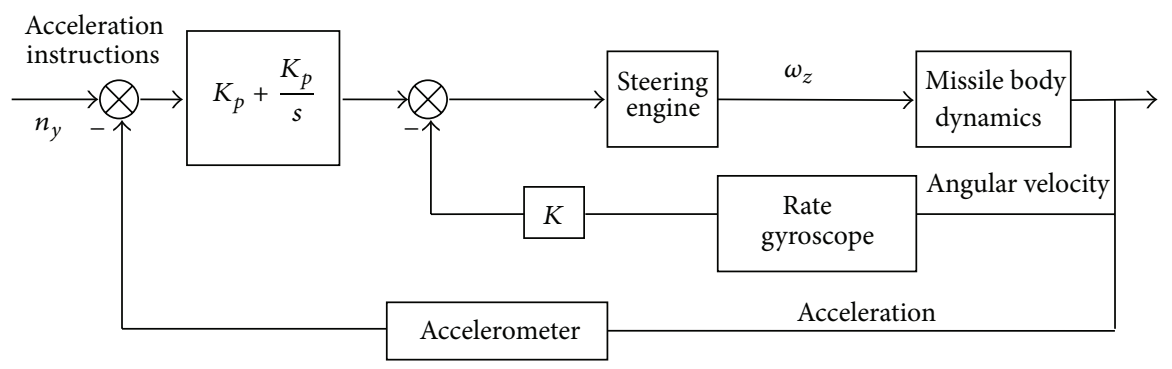

FIGURE 2: Basic structure of pitch channel autopilot.

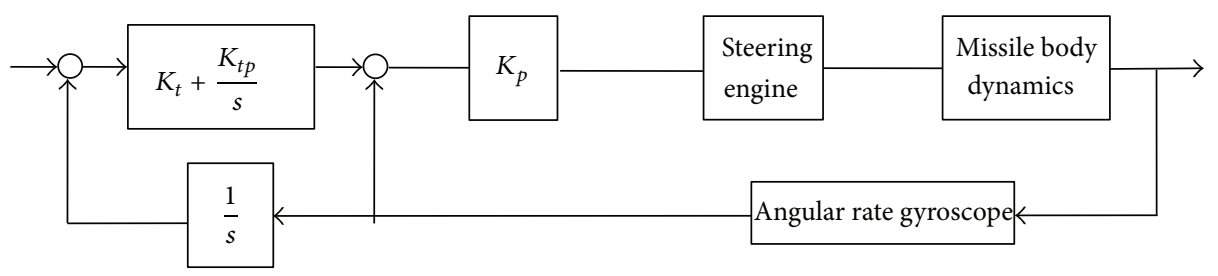

Figure 3: Basic structure of rolling channel autopilot.

the guidance control instruction, and it consists of angular rate gyro feedback loop and the accelerometer feedback loop. Angular rate gyro feedback loop can realize artificial stabilization of missile body. The accelerometer feedback loop can control missile normal overload by PI. The solution has the following characteristics:

(1) angular rate gyro composes damping feedback loop and is used to adjust damping characteristics and system needed, increase equivalent damping of missile, and improve the system bandwidth and its dynamic quality;

(2) overload obtained by accelerometer composes main feedback loop structure, achieves ratio relation between steady normal overload and control instruction, guarantees the steady-state accuracy of overload control missile, and improves the ability of antijamming.

Pitch channel autopilot basic structure is shown in Figure 2 .

(2) Yaw Channel Autopilot. The yaw channel autopilot is similar to pitch channel autopilot.
(3) Rolling Channel Autopilot. Roll channel autopilot is used for stable rolling angle position and rolling speed of missile, requires quite stability margin and enough rapidity, in order to eliminate rolling moment caused by effect of interference torque in the missile flight, and tries to reduce the static error of tilt circuit; missile roll channel autopilot adopts the roll angle and roll angle rate feedback structure.

Rolling channel autopilot basic structure is shown in Figure 3.

2.4. Controller Parameters Design. Consider control system parameters design of pitch channel.

Pitch channel parameters design is shown in Figure 4. In the simulation model, FinActuator stands for steering engine model, Transfer Fun2 stands for missile body linear transfer function, and Transfer Funl stands for missile body transfer function from pitching angle rate to normal overload. First, use classical control theory design method, design the inner loop angular rate feedback loop gain coefficient $K$ _inner, make the circuit with suitable damping, and then design PI controller parameters the outer loop normal overload loop and design the parameters $K_{i}$ and $K_{p}$ using the pole assignment method. 


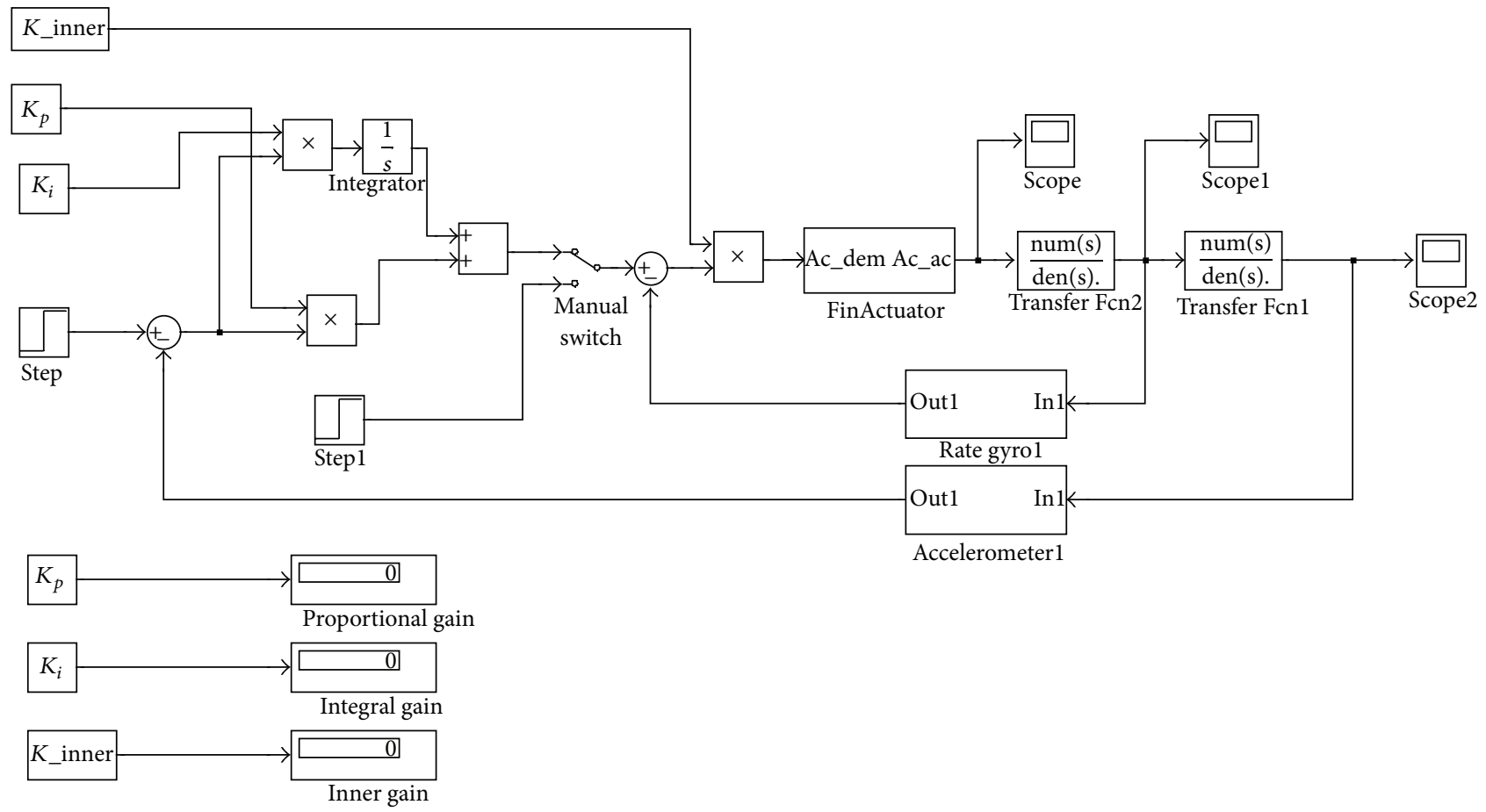

Figure 4: Parameters design of pitch channel.

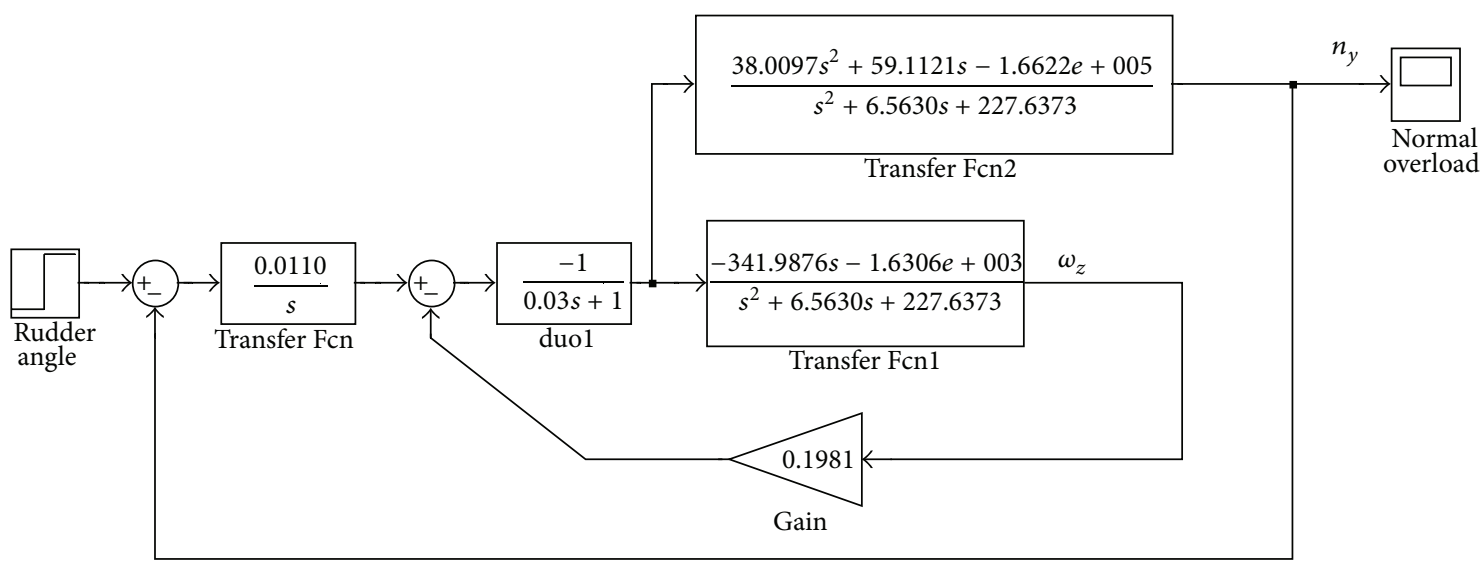

FIGURE 5: Controller structure of pitch channel.

Controller structure of pitch channel and rolling channel is shown in Figures 5 and 6 ; the value of parameters can be computed by fundamental formulas $a_{i}, c_{i}$ which has been defined above through engineering method.

\section{Realization of System Simulation Model}

3.1. Six-Degree-of-Freedom Simulation Model of Guidance and Control System. Composition of missile guidance control system includes the missile body dynamics/kinematics module, stable algorithm module, guidance law module, seeker module, servo module, target motion module, and missiletarget relative kinematics module.
Guidance system and control system are the important parts in missile system, and it is the key to missile final precision and system performance. Missile circuit design is mainly the double circuit design of control loop and guidance loop; the missile guidance control system design is the key part in the missile design process $[12,13]$.

For the actual missile, there are different constraints on other aspects in addition to ensuring the accuracy of hitting target and motor overload due to different geometry design and operational requirements in the guidance control law design, such as big attack angle constraint, big landing angle constraint when being against ground targets, field-of-view angle constraint, speed constraint, and other constraints. So, full consideration should be given to the requirements of 


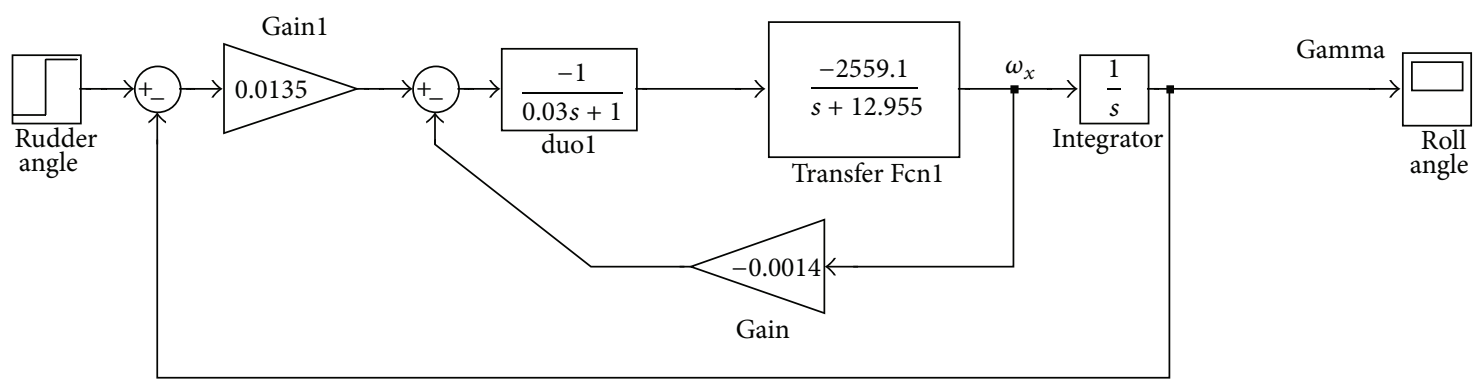

FIgURE 6: Controller structure of rolling channel.

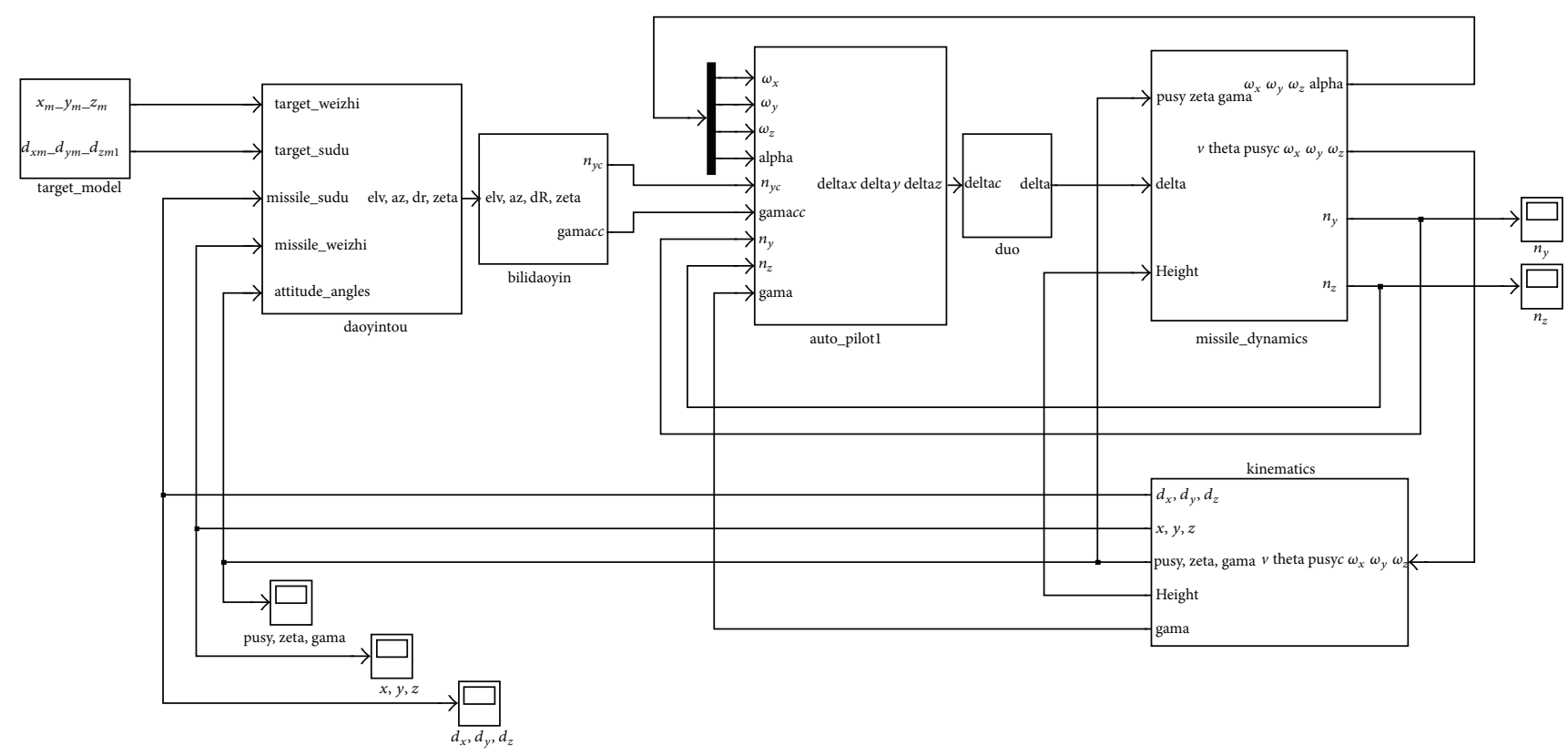

FIGURE 7: Relationship between all simulation modules.

these constraints in the guidance law and control law design progress. Usually, the steps of missile guidance and control system integrated design are as follows:

(1) analyze tactical technical assignment and design scheme of missile and determine the various constraint conditions of missile guidance and control system;

(2) consider six-degree-of-freedom modeling according to the missile aerodynamic parameters and guidance mode;

(3) design appropriate guidance law according to restrictions of missile guidance law and guidance simulation for particle object;

(4) design appropriate control law according to restrictions of missile control law and simulation for control system;

(5) build six-degree-of-freedom missile model and bring guidance law and control law into simulation and then adjust parameters;
(6) change the parameters of missile and interference, examine the stability of the missile guidance control system, and determine the guidance precision of missiles and other indicators through the multiple target simulation with different initial conditions.

3.2. Simulink Simulation Realization. Using Simulink simulation, encapsulate six submodules which are, respectively, "target motion module," "seeker module," "proportional guidance module," "autopilot module," "module projectile movement," and "moving target tracking module" and then connect each module, debugging and operation, and realize the simulation of the missile $[14,15]$. Relationship between all simulation modules is shown in Figure 7.

The target movement information generated by target motion module, target maneuvers in a two-dimensional plane, and seeker module measures target location and calculates angle and position relationship between missile and target location combined with the missile's position. 


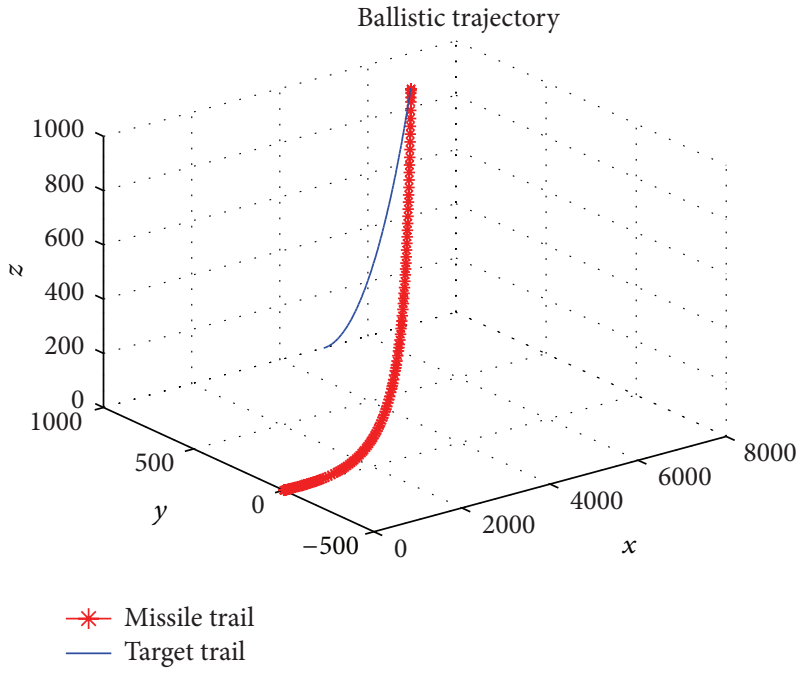

FIgURE 8: Ballistic trajectory.
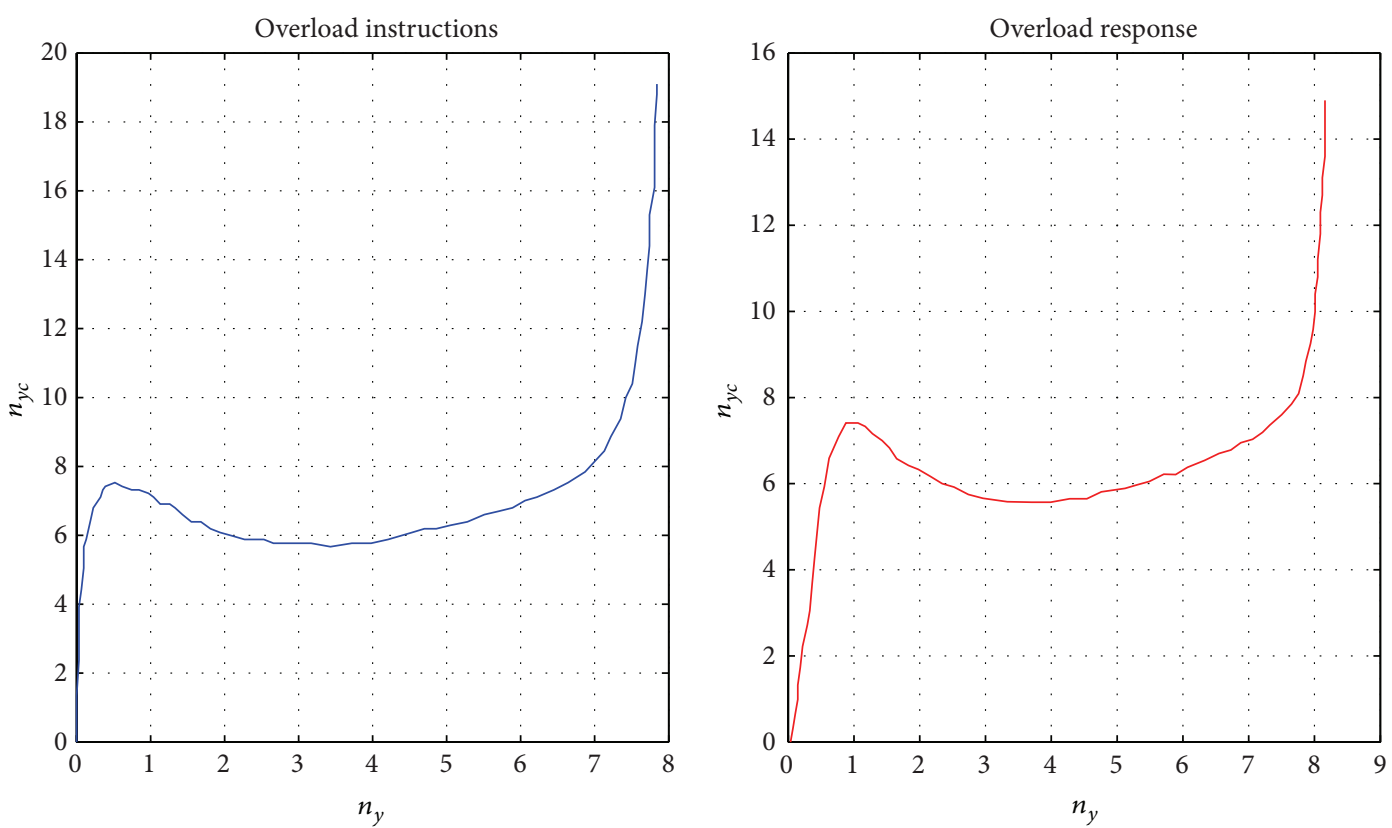

FIGURE 9: Overload instructions and missile body overload response.

The get_elv_az_dr module is the core part of calculating missile-target angle relationship, used to calculate relatively the position of missile-target.

There is a control module for stopping simulation in seeker module; that is, when missile-target distance is less than 10, affirm that the missile has hit the target and stop simulation.

3.3. Large System Simulation. Large system Simulink simulation time length is $8.15 \mathrm{~s}$; the ballistic trajectory simulation result is shown in Figure 8.

Overload instructions and missile body overload response presented through missile guidance law in the process of missile flight simulation are shown in Figure 9.

\section{Conclusions}

This paper introduces the engineering method of building missile six-degree-of-freedom model, steps of missile guidance, and control loop general design method as follows: first, it proposes building missile six-degree-of-freedom Simulink model and gives a brief introduction of the working process of the simulation model. Secondly, it presents control gain step response design and the results of pitch channel, yaw channel, and roll channel of simplified missile body. Finally, it concludes the Simulink simulation of trajectory attack process, missile's attitude angle, normal overload command, and normal overload response variation. 
From the digital simulation and analysis, the following conclusions can be obtained.

(1) From the perspective of Simulink simulation, the missile guidance control system works properly under the effect of controller parameters designed, precise tracking, and hitting a target moving at a constant speed. In this case, the target must be moving within a two-dimensional plane and the missile's overload and attitude angle must remain within a reasonable range conforming to actual situation.

(2) It can be seen from the step response results of pitch channel and rolling channel that the design of the controller gain is appropriate according to the simplified missile model. Relative to controller of each channel gain, step response of simplified missile body is floating, small overshoot and adjustment time, and it is in the reasonable range.

(3) There are some problems that need to be further solved in the Simulink simulation; for example, it does not take the effect of noise influence on guidance error in guidance and control system into account, and without considering disturbance rejection.

(4) Later we can try to design system bandwidth using correction network so as to avoid noise and we can convert the missile to generalized object analysis performance of missile guidance and control system and then we can also analyze robust stability and robust performance of uncertainty of missile body parameters.

\section{Conflict of Interests}

The authors declare that there is no conflict of interests regarding the publication of this paper.

\section{References}

[1] J.-F. Cossette, P. K. Smolarkiewicz, and P. Charbonneau, "The monge-ampère trajectory correction for semi-lagrangian schemes," Journal of Computational Physics, vol. 274, pp. 208229, 2014.

[2] G. Buonincontri, C. Methner, T. Krieg, T. A. Carpenter, and S. J. Sawiak, "Trajectory correction for free-breathing radial cine MRI," Magnetic Resonance Imaging, vol. 32, no. 7, pp. 961-964, 2014.

[3] X. Bin and Z. Liang, "Trajectory correction projectile and analysis on the key technologies for the trajectory correction process," National Defense Science \& Technology, vol. 34, no. 3, pp. 27-33, 2013.

[4] M. Martínez, N. Bruno, and R. Kelly, "Ballistic and weaponeering analysis of projectiles using optical strobe tracer as selfregistration method to improve accuracy and crease dispersion of direct and indirect fire weapons," in Proceedings of the 27th International Symposium on Ballistics, pp. 302-313, Feriburg, Germany, April 2013.

[5] S. Qiang, Z. Pian, Y. Deng-Hong, and L. Dong-Guang, "Correction strategy analysis on one-dimensional trajectory correction fuze," Transactions of Beijing Institute of Technology, vol. 33, no. 5, pp. 445-448, 2013.
[6] X. Yi-Da, "Intellgent munitions in China: China has developed advanced TSMs in the world," Chinese Journal of Energetic Materials, vol. 19, no. 3, pp. 251-255, 2011.

[7] X.-F. Sun, L.-X. Gu, and C.-L. Gong, "Dynamics of a deflectablenose missile," Science China Technological Sciences, vol. 55, no. 12, pp. 3483-3494, 2012.

[8] X.-F. Sun, L.-X. Gu, and C.-L. Gong, "Overload control system design of a deflectable nose missile," Procedia Engineering, vol. 15, pp. 402-407, 2011.

[9] G. Yuan, G. Liang-Xian, and P. Lei, "Modeling and simulating dynamics of missiles with deflectable nose control," Chinese Journal of Aeronautics, vol. 22, no. 5, pp. 474-479, 2009.

[10] N. F. Palumbo, B. E. Reardon, and R. A. Blauwkamp, "Integrated guidance and control for homing missile," Johns Hopkins APL Technical Digest, vol. 25, no. 2, pp. 121-139, 2004.

[11] P. Wernert and S. Theodoulis, "Modelling and stability analysis for a class of $155 \mathrm{~mm}$ spin-stabilized projectiles with course correction fuse," in Proceedings of the AIAA Atmospheric Flight Mechanics Conference, 2011.

[12] G. A. Korn, "Continuous-system simulation and analog computers: from op-amp design to aerospace applications," IEEE Control Systems Magazine, vol. 25, no. 3, pp. 44-51, 2005.

[13] S. Theodoulis, V. Gassmann, T. Brunner, and P. Wernert, "Robust bank-to-turn autopilot design for a class of $155 \mathrm{~mm}$ spin-stabilized canard-guided projectiles," in Proceedings of the AIAA Atmospheric Flight Mechanics Conference (AFM '13), 2013.

[14] X. Ming-You, Advanced Exterior Ballistics, Advanced Education Press, Beijing, China, 2003.

[15] H. Zi-Peng, External Ballistics, National Defence Industry Press, Beijing, China, 2000. 


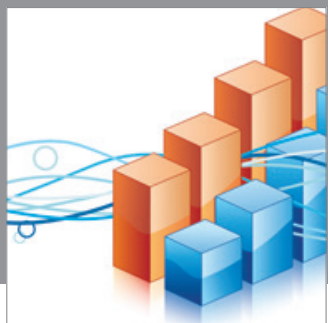

Advances in

Operations Research

mansans

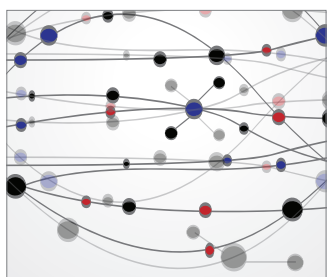

The Scientific World Journal
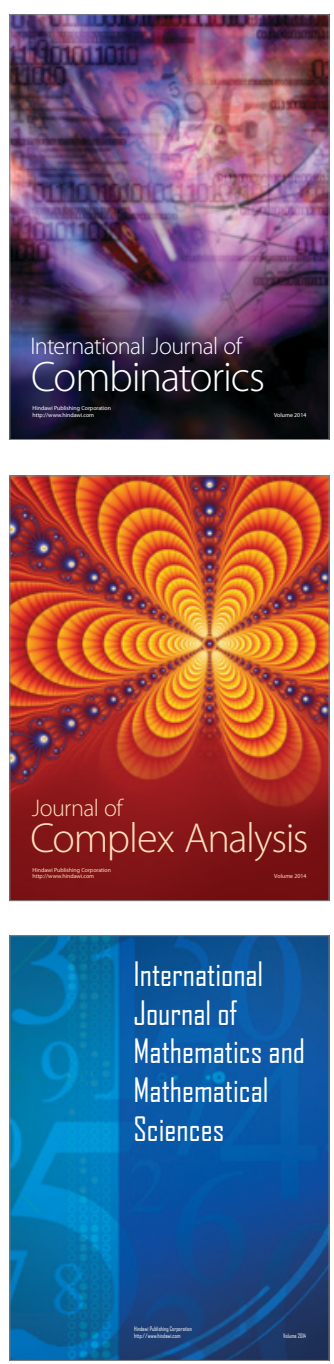
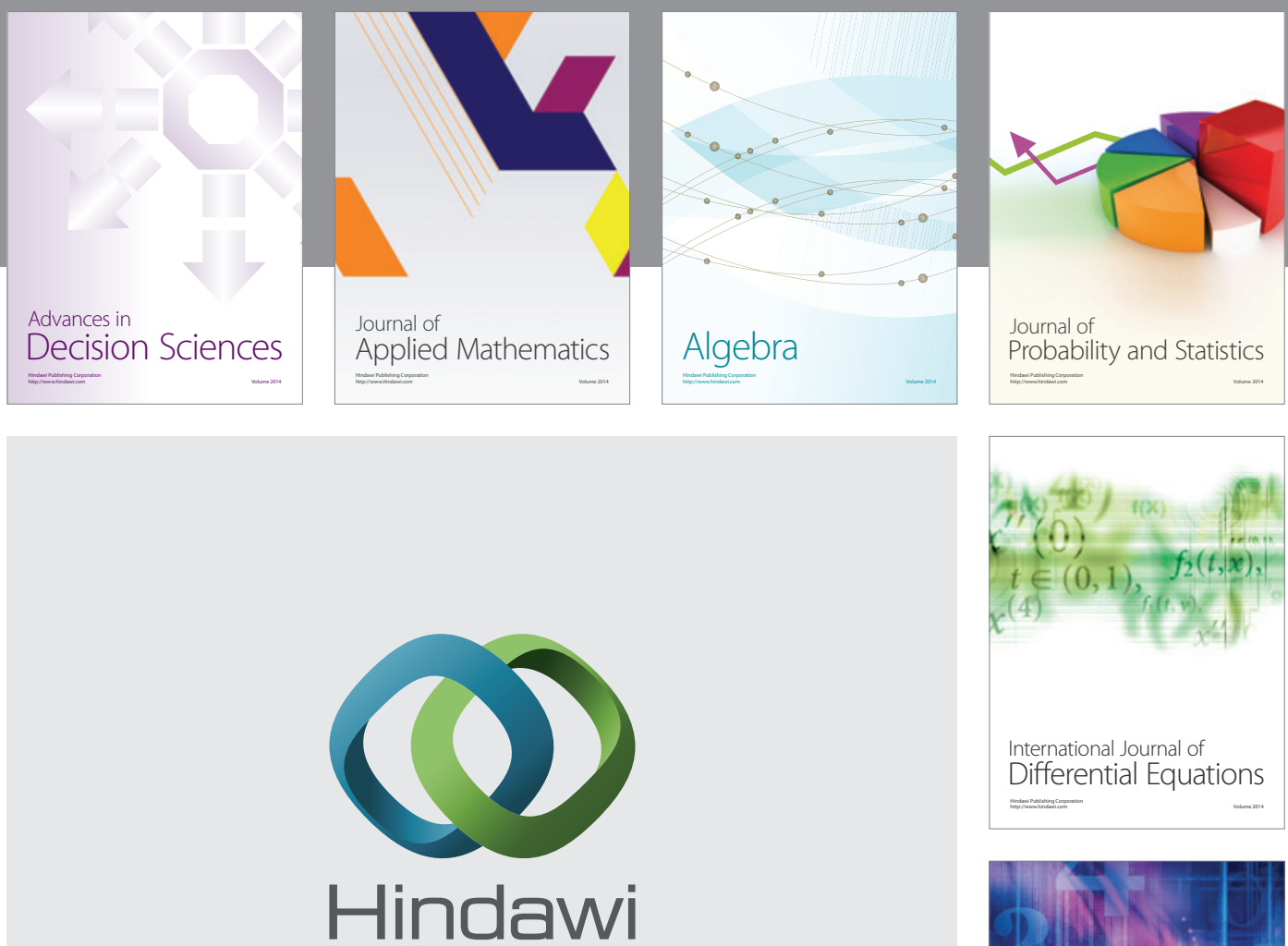

Submit your manuscripts at http://www.hindawi.com
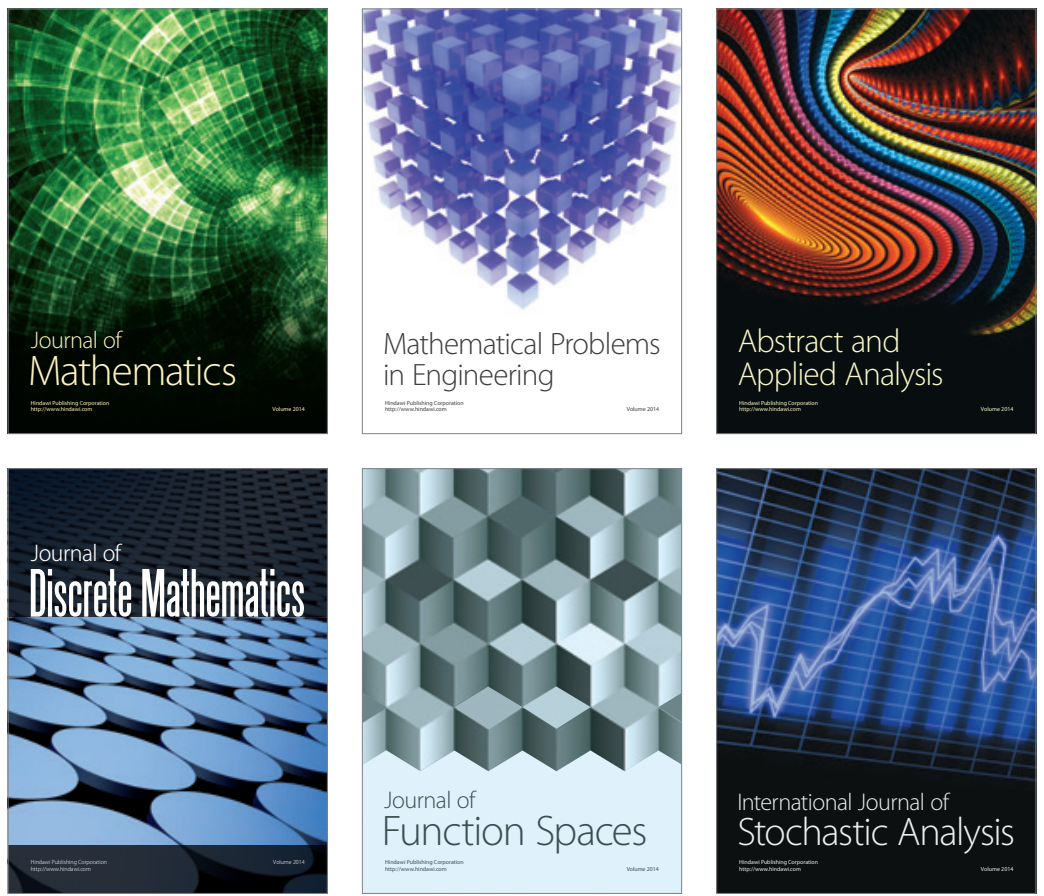

Journal of

Function Spaces

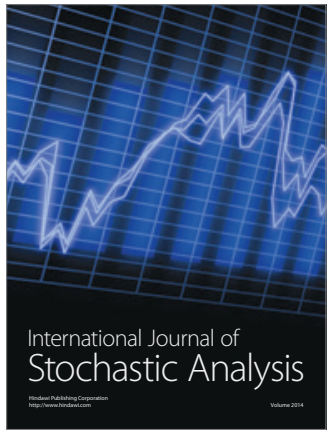

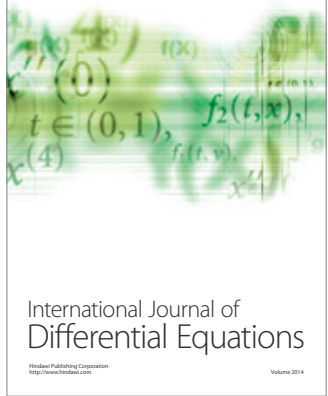
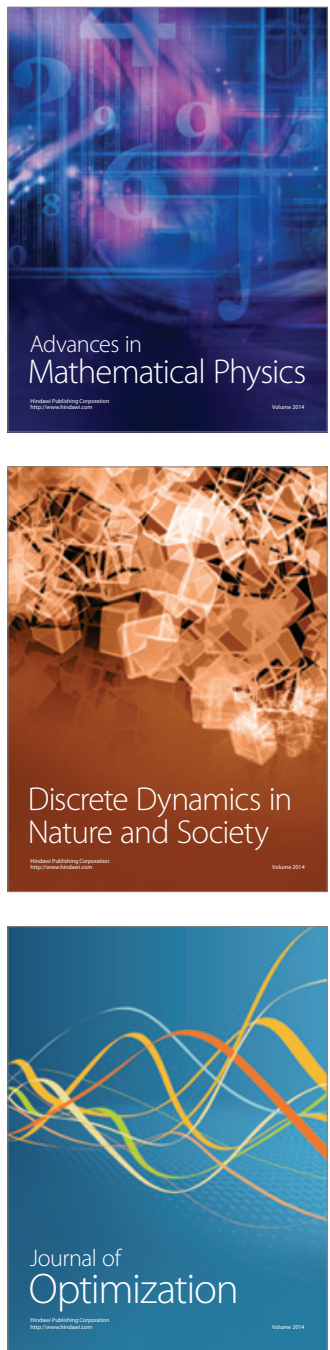